D) Check for updates

Cite this: Mater. Adv., 2022,

3,2692

Received 22nd November 2021

Accepted 29th January 2022

DOI: $10.1039 / \mathrm{d} 1 \mathrm{ma} 01100 f$

rsc.li/materials-advances

\section{Study on the different mechanoluminescence of anthracene derivatives $\uparrow$}

\author{
Jun Miao, $\ddagger$ Zhaoxia Zhang, $\$$ Zhiyuan Cui and Ming Zhang (D) *
}

The mechanoluminescence (ML) properties of anthracene derivatives were studied. The fountainhead molecule is a plain compound BN, consisting of anthracene and boric acid ester. Then, by introducing three simple substituent groups to $\mathrm{BN}$, three derivatives (BO, $\mathrm{BE}$ and $\mathrm{BB}$ ) were obtained to adjust the packing modes and intermolecular interactions of the molecules simultaneously. Among these four compounds, BN presented bright blue ML observed under daylight and could maintain ML for more than 2 min by constant grinding. The molecule BO substituted by an electronwithdrawing nitrobenzene group showed green weak ML. However, the molecules $B E$ substituted by an electron-withdrawing formyl unit and BB substituted by an electron-donating diphenylamine unit displayed no ML. Furthermore, according to the crystal analysis and DFT calculations, the preliminary relationship between the ML properties and molecular structure was summarized. A feasible design strategy of anthracene derivatives with $M L$ is proposed.

\section{Introduction}

Mechanoluminescence (ML) or triboluminescence (TL), which is induced by mechanical force on crystals or microcrystals without UV irradiation, was first found by Francis Baon in $1605 .{ }^{1-3}$ And the first TL spectrum was reported by Longchambon in 1922 from sucrose. ${ }^{4}$ Since then, many attempts have been made to describe its nature. So far, though the ML phenomenon has been found for more than 400 years, there is still not a universal, systematic and clear theory to clarify the entire ML phenomenon. This is because the ML process occurs too fast to be observed and so many unknown factors limit the detailed and deep investigations. Encouragingly, it is certain

State Key Laboratory of Supramolecular Structure and Materials, College of Chemistry, Jilin University, Changchun 130012, P. R. China.

E-mail: zhming@jlu.edu.cn

$\dagger$ Electronic supplementary information (ESI) available. CCDC 2048014-2048016 and 2089171. For ESI and crystallographic data in CIF or other electronic format see DOI: $10.1039 / \mathrm{d} 1 \mathrm{ma} 01100 \mathrm{f}$

\# Jun Miao and Zhaoxia Zhang contributed equally to this work. that the ML properties are closely related to the crystal structures of molecules, and piezoelectric crystals are more likely to exhibit ML due to the charges accumulated on the crack surface, which leads to intense electron bombardment and then produces luminescence. ${ }^{5-9}$ These points are still supported by current studies and many interesting studies have been carried out. In 1922, Longchambon also concluded that noncentrosymmetric crystals were more inclined to present ML. ${ }^{10}$ In 2012, Nakayama and his colleagues designed a series of $\mathrm{N}$ phenyl-substituted imides and concluded that a dipolar molecular structure and non-centrosymmetric arrangement in the crystals played a key role in ML. ${ }^{11}$ In 2015, Chi et al. introduced the concept of AIE (aggregation-induced emission) to ML for the first time, which proposed a new design idea and opened up a new research perspective for the further development of ML. ${ }^{12}$ In 2019, Li et al. showed that the introduction of different halogen atoms may lead to different intermolecular interactions, and efficient intermolecular interactions can reduce the non-radiative energy loss and contribute to the ML properties. ${ }^{13}$ After that, a pure hydrocarbon tetraphenylethene (TPE) derivative with ML, TETPE, was reported in $2017 .{ }^{14}$

In a previous report, we investigated the effects of changing the linkage site of thiophene and introducing methyl groups in thiophene on the ML properties of anthracene derivatives. ${ }^{15}$ However, in this paper, we introduce different groups to explore the factors affecting the $\mathrm{ML}$ of anthracene derivatives, broaden the variety of anthracene ML materials, and contribute to our better understanding of ML. Here, we report an anthracene derivative BN with a bright ML even in daylight, and theML of BN can be maintained for a long time even under continuous stimulation. Compared with the previously reported thiophene derivatives, the compound BN synthesized in this paper has a simple synthesis procedure and a strong ML phenomenon under sunlight, which is more likely to be applied in practice. ${ }^{16,17}$ Then, by introducing different substituent groups, the effects of the conformation, the molecular packing modes and the intermolecular interactions on the ML properties were investigated. The results show that the molecular packing 
modes and suitable intermolecular interactions play a key role in the ML properties of anthracene derivatives.

\section{Results and discussion}

\section{The synthesis and the optical properties}

Here, anthracene was selected as a simple condensed aromatic molecule due to its high photoluminescence (PL) efficiency. ${ }^{18,19}$ But its stronger intermolecular $\pi-\pi$ stacking weakens its PL intensity in the solid state, and thus the fountainhead compound BN was synthesized by introducing the boric acid ester into the "9" site of anthracene to reduce the $\pi-\pi$ stacking. In addition, the linkage of a borate ester group can enhance the effective intermolecular interactions, which can reduce the energy loss of the non-radiative relaxation during mechanical stimulation and facilitate the achievement of ML. ${ }^{20,21}$ Then, a nitrobenzene/formyl group as an electron-withdraw group and diphenylamine as an electron-donating group were linked to the site " 10 " of $\mathrm{BN}$ to obtain compound $\mathrm{BO}, \mathrm{BE}$ and $\mathrm{BB}$, respectively (Fig. 1a). The detailed syntheses and characterizations of these four compounds are presented in Scheme S1 (ESI $\dagger$ ).

The optical properties of these four anthracene derivatives were studied. It can be seen from Fig. S1 (ESI $\dagger$ ), that the absorption peaks of $\mathrm{BN}$ in THF $\left(10^{-5} \mathrm{M}\right)$ are located at $253 \mathrm{~nm}, 345 \mathrm{~nm}, 363 \mathrm{~nm}$ and $382 \mathrm{~nm}$, which belong to the $\pi-\pi^{*}$ transition of the anthracene moiety. ${ }^{22,23}$ The introduction of nitrobenzene, formyl and diphenylamine groups results in a red-shift of the absorption spectra compared to $\mathrm{BN}$, which is due to the increased conjugation of the molecules. The PL spectra of BN, BO, BE and BB in THF are collected in Fig. 1b. It can be seen that the maximum emission peak of $\mathrm{BN}$ is at $425 \mathrm{~nm}$, while the maximum emissions of $\mathrm{BO}, \mathrm{BE}$ and $\mathrm{BB}$ are at $545 \mathrm{~nm}, 430 \mathrm{~nm}$ and $510 \mathrm{~nm}$. Compared with BN, the emission spectra of $\mathrm{BO}, \mathrm{BE}$ and $\mathrm{BB}$ show different degrees of red-shift, which is also due to the introduction of different groups to increase the conjugation of the molecules. (a)

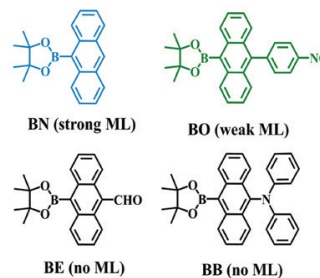

(c)

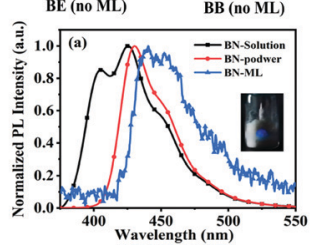

(b)

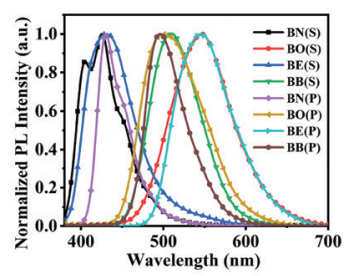

(d)

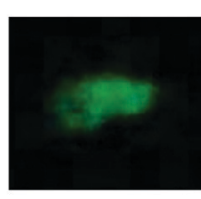

Fig. 1 (a) The chemical structures of $B N, B O, B E$ and BB. (b) The normalized PL spectra of $B N, B O, B E$ and $B B$ in THF (s) and their powders $(P)$. (c) The PL of BN in different states and the ML of BN. The inset: Photo of the BN's ML under daylight (d) photo of BO's ML in the dark.
Moreover, their PL quantum yields (PLQY, $\Phi_{\mathrm{F}}$ ) and the PL lifetimes $(\tau)$ in THF solution were also tested and summarized in Table S1 (ESI $\dagger$ ). The PLQYs of BN, BO, BE and BB are $36.77 \%$, $9.48 \%, 0.09 \%$ and $55.76 \%$, respectively. From the above results, it can be found that for PLQY, the introduction of an electronwithdrawing group to $\mathrm{BN}$ has negative effects, while the introduction of an electron-donating group to $\mathrm{BN}$ has positive effects: $\mathrm{BO}$ and BE present lower PLQY, while BB shows the highest PLQY. The introduction of diphenylamine increases the degree of electron conjugation, leading to enhanced fluorescence, while the introduction of nitro and formyl groups also expands the extent of electron conjugation, resulting in fluorescence red-shift but leading to a weakening of the fluorescence intensity. This is because the introduction of nitro and formyl groups will normally promote excitons from the singlet excited state through intersystem crossing to the sensitive triplet state, and these excitons will be dissipated through non-radiative channels, leading to diminished fluorescence. ${ }^{24,25}$

Furthermore, their PL behaviors in the powder state were carefully investigated. As shown in Fig. 1b, the maximum PL peak of the $\mathrm{BN}$ powder is at $431 \mathrm{~nm}$, and the maximum $\mathrm{PL}$ peaks of $\mathrm{BO}, \mathrm{BE}$ and $\mathrm{BB}$ powders are at $500 \mathrm{~nm}, 545 \mathrm{~nm}$ and $495 \mathrm{~nm}$, respectively. Compared with $\mathrm{BN}$ powder, the $\mathrm{BO}, \mathrm{BE}$ and $\mathrm{BB}$ powders present different degrees of red-shift in the PL spectra, which is due to their enhanced conjugation. In addition, the PLQY and the PL lifetimes $(\tau)$ of their powders were also investigated (Table S1, ESI $\dagger$ ). The same results as those in the solution can be reached.

\section{The ML properties of $\mathrm{BN}, \mathrm{BO}, \mathrm{BE}$ and $\mathrm{BB}$}

Among these four compounds, BN shows the strongest ML properties, which can even be observed under daylight (the inset of Fig. 1c). What's more, the ML of BN can still be observed after continuous grinding for more than 2 minutes (Video S1, ESI $\dagger$ ). It can be seen from Fig. 1c that the ML maximum emission peak of $\mathrm{BN}$ is located around $440 \mathrm{~nm}$, which presents a $9 \mathrm{~nm}$ red-shift compared to its powder $(431 \mathrm{~nm})$. As we know that ML and PL have different excitation processes, PL is generated by the excitation of surface molecules, while ML occurs at cracks in the crystal. The ML light must pass through the crystal and will be partially absorbed, which causes the ML to be shifted to a slightly longer wavelength. ${ }^{26}$

The molecule of $\mathrm{BO}$ substituted by electron-withdrawing nitrobenzene presents faint green ML in the dark, which is too weak to be recorded (Fig. 1d). And the ML of BO could not be maintained for a long time under continuous external forces. However, the ML phenomenon could not be observed in BE substituted with a formyl group and in BB substituted with diphenylamine. From the above results, it can be found that both electron-donating substituents and electronwithdrawing substituents are detrimental to the ML, but they can adjust the color of the ML. Therefore, these different ML properties are most probably related to molecular packing modes and intermolecular interactions.

\section{The relationship between packing mode and ML}

The single crystals of the four anthracene boronic ester derivatives were obtained by slow evaporation from mixed solvent 
(dichloromethane and ethanol) at room temperature. The single crystal X-ray diffraction was carried out on BN, BO, BE and $\mathrm{BB}$ to investigate the relationship between packing modes and ML. The CCDC numbers of $\mathrm{BN}, \mathrm{BO}, \mathrm{BE}$ and $\mathrm{BB}$ are 2048014, 2048016, 2048015 and 2089171, respectively. We have published the crystallographic data and the ESI $\dagger$ data (Table S7, ESI $\dagger$ ) for the structures reported in this paper as CDC Communication articles in the Cambridge Crystallographic Data Centre. $^{27-30}$

In $\mathrm{BN}$ crystals, the adjacent molecules exhibit a stuck packing mode, while in $\mathrm{BO}, \mathrm{BE}$ and $\mathrm{BB}$ crystals, the adjacent molecules are arranged parallel to each other and show a parallelogram packing mode (Fig. 2). The stuck packing mode in crystals can limit the molecular slipping under the action of external forces to a certain extent, which makes the energy loss of non-radiative transitions less and facilitates the realization of bright ML. However, the parallelogram packing mode is prone to molecular slippage under the action of external forces, leading to the loss of the non-radiative transition energy, which is not conducive to the ML phenomenon.

Moreover, the space groups of $\mathrm{BN}, \mathrm{BO}, \mathrm{BE}$ and $\mathrm{BB}$ are $P 2_{1}$, $P 1, P 2_{1} / c$ and $P \overline{1}$, respectively. The space groups of $\mathrm{BB}$ and $\mathrm{BE}$ crystals without $\mathrm{ML}$ are centrosymmetric, whereas the crystal space groups of $\mathrm{BN}$ with strong $\mathrm{ML}$ and $\mathrm{BO}$ with weak ML are non-centrosymmetric, which means that they have piezoelectric effects in the crystal. For piezoelectric crystals, the accumulation of charges on the cracked surface creates a strong electric field to excite the light. ${ }^{31-34}$ Based on these results, it can be found that among the affecting factors, the piezoelectric effect originating from the non-centrosymmetric space group plays a more positive role for ML. Besides, the stuck packing mode plays an important role in achieving ML properties. Thus, due to the stuck packing modes and piezoelectric effects, BN presents a stronger ML emission intensity.

\section{The relationship between intermolecular interactions and ML}

Besides the piezoelectric effect, the efficient intermolecular interactions also play an essential role in the ML properties, which can restrain the non-radiative transition process under mechanical force and be more favorable to the ML generation. To analyze and investigate the relationship between ML and intermolecular interactions, the minimum non-repeating units (BN, BO, BE and $\mathrm{BB}$ are eight molecules) were selected as the research objects. It should be noticed that only the intermolecular interactions shorter than $4 \AA$ in these single crystals are considered here (in the dashed area in Fig. 2).

As shown in Fig. 3 and Fig. S3, Tables S2 and S3 (ESI $\dagger$ ), the BN crystal with strong ML contains $43 \mathrm{C}-\mathrm{H} \cdots \pi$ interactions $(2.859 \AA-3.993 \AA)$, and $12 \mathrm{C}-\mathrm{H} \cdots \mathrm{O}$ interactions $(2.611 \AA$ $3.978 \AA)$. The weak ML BO crystals include $58 \mathrm{C}-\mathrm{H} \cdots \pi$ interactions (2.603 $\AA-3.985 \AA)$, and $28 \mathrm{C}-\mathrm{H} \cdots \mathrm{O}$ interactions (2.720 ̊-3.850 ̊). In general, strong intermolecular interactions will cause the molecules to stack more tightly and result in high crystal rigidity, which can avoid the energy loss caused by the possible molecular slippage under mechanical forces, and suppress the non-radiative transition process under mechanical stimulation, which is more advantageous to ML. ${ }^{7,13,35,36}$ The intermolecular interactions in the BO crystals are stronger than those in $\mathrm{BN}$, but exhibit weak ML properties. This may be because the intermolecular interactions in the crystal are too strong, which makes the crystal too rigid to be broken by external forces, and results in unsatisfactory ML
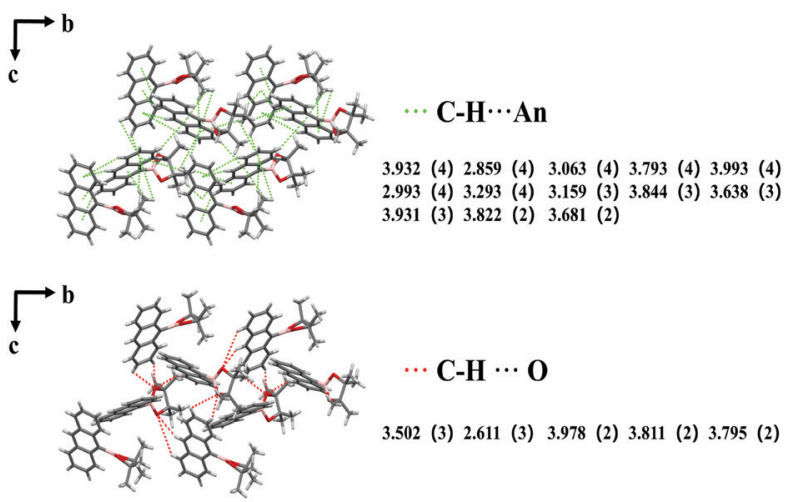

Fig. 3 The intermolecular interactions including $\mathrm{C}-\mathrm{H} \cdots \pi$ (green) and $\mathrm{C}-\mathrm{H} \ldots \mathrm{O}$ (red lines) in the BN crystal (eight molecules).
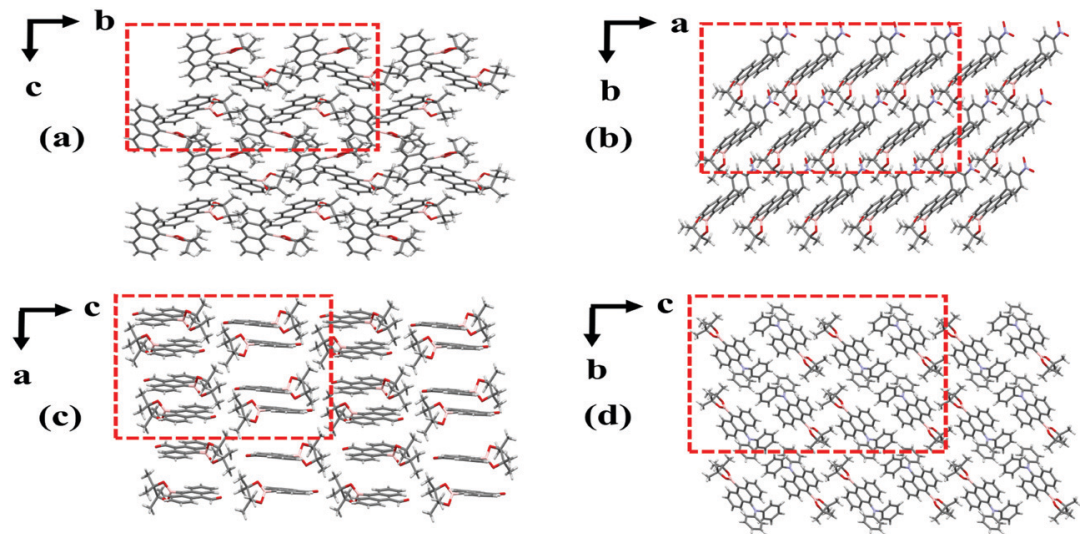

Fig. 2 Packing modes of the BN (a), BO (b), BE (c) and BB (d) crystals with different directions. The minimum non-repeating unit in the dashed area. 
performance. From the above results, it can be seen that intermolecular interactions affect the ML properties. The strong intermolecular interactions are beneficial for ML, which means that the ML can be maintained for a long time under the action of external forces. However, this does not mean that the stronger the intermolecular interactions are, the stronger the $\mathrm{ML}$ is, and appropriate intermolecular interactions are needed.

\section{Powder X-ray diffraction (PXRD)}

To further understand the factors affecting ML, powder X-ray diffraction (PXRD) was performed on powders of BN and BO with ML properties. As can be seen in Fig. S6 (ESI $\dagger$ ), the diffraction peaks in the powder XRD pattern are similar to the single crystal simulated PXRD pattern, which indicates that the powder and single crystal have similar packing modes. The as-prepared samples $\mathrm{BN}$ and $\mathrm{BO}$ exhibit sharp peaks in the PXRD pattern, which discloses their good crystallinity. After being ground lightly (the force was around $1 \mathrm{~N}, 1 \mathrm{~min}$ ), the diffraction peaks of $\mathrm{BN}$ and $\mathrm{BO}$ still exhibit sharp and high diffraction peaks; then, after being ground heavily (the force was around $2 \mathrm{~N}, 1 \mathrm{~min}$ ), the diffraction peaks only show a little decrease. These results indicate that the BN and BO crystals have high lattice stability and the stacking pattern is not easily disrupted, which is beneficial for ML realization.

From the results of crystal analysis and PXRD, it can be seen that the stuck packing mode, the moderate intermolecular interaction and the high lattice stability in the BN crystals can avoid the energy loss caused by the possible molecular slip under mechanical forces, inhibit the non-radiative transition process under mechanical stimuli, and facilitate the ML for a long time under continuous mechanical stimulation. However, for BO crystals, firstly, the intermolecular interactions are stronger than those of BN, resulting in higher crystal rigidity, which is difficult to destroy under the action of external forces. Secondly, the molecules form the parallelogram stacking modes in the BO crystals, which are prone to molecular slippage under the stimulation of external forces, then leading to the energy loss of non-radiative transition, which is not conducive to the ML. Thus, these multiple factors lead to the weak ML of BO.

\section{Theoretical calculations}

Density functional theory (DFT) calculations (B3LYP/6-31g(d,p)) were carried out on the isolated molecules (derived from their ground state geometries in single crystals). As shown in Fig. S7 (ESI $\dagger$ ), it can be found that the substituent groups have a significant effect on the HOMO and LUMO, which then leads to the different optical properties of $\mathrm{BN}, \mathrm{BO}, \mathrm{BE}$ and $\mathrm{BB}$ to a great extent.

Commonly, a non-centrosymmetric crystal with a larger dipole moment will produce a stronger piezoelectric effect. $^{17,37}$ The dipole moment of the non-centrosymmetric crystal BO is 8.48 Debye, while the dipole moments of $\mathrm{BN}$ are only 2.75 and 2.71 Debye. According to the literature, the net dipole moment of the coupled molecules can explain the ML properties. ${ }^{38-41}$ Therefore, the coupled molecules with strong intermolecular interactions in the $\mathrm{BN}$ crystals were selected for DFT calculations (B3LYP/6-31g(d,p)). According to Fig. S8 (ESI $\dagger$ ), the net dipole moment of the coupled molecules is larger than that of isolated molecules. The large net dipole moment of the non-centrosymmetric BN crystal can accumulate more charges on the crack surface under the effect of mechanical stimulation, and the strong electric field formed will act as an excitation source to generate ML, which has an important contribution to its ML.

\section{Conclusions}

Here, we reported a series of anthracene boronic ester derivatives with different ML activities. Furthermore, the factors that affect their ML properties were carefully investigated. According to the results, the change of electron density caused by different substituents mainly adjusted the fluorescence and ML color of the material. The molecular packing modes and intermolecular interactions are the most important factors for ML. Moreover, though the strong intermolecular interactions in crystals can reduce the energy loss of non-radiative transitions under mechanical stimulation and benefit the bright ML, it is not implied that the stronger the intermolecular interactions are, the stronger the ML will be, and appropriate intermolecular interactions are necessary for the achievement of the ML. Hereby, these research results help us to better understand and apply ML materials to so degree.

\section{Conflicts of interest}

There are no conflicts to declare.

\section{Acknowledgements}

We are grateful for the financial support from the National Natural Science Foundation of China (Grant No. 21875083) and Supported by the Program for JLU Science and Technology Innovative Research Team (JLUSTIRT program, Grant No. 2019TD-33).

\section{Notes and references}

1 P. Jha and B. P. Chandra, Luminescence, 2014, 29, 977-993.

2 B. P. Chandra, V. K. Chandra, P. Jha, R. Patel, S. K. Shende, S. Thaker and R. N. Baghel, J. Lumin., 2012, 132, 2012-2022.

3 M. Fang, J. Yang, Q. Liao, Y. Gong, Z. Xie, Z. Chi, Q. Peng, Q. Li and Z. Li, J. Mater. Chem. C, 2017, 5, 9879-9885.

4 B. P. Chandra, V. K. Chandra and P. Jha, J. Lumin., 2013, 135, 139-153.

5 A. Chakravarty and T. E. Phillipson, J. Phys. D: Appl. Phys, 2004, 37, 2175-2180.

6 B. P. Chandra, S. K. Mahobia, P. Jha, R. K. Kuraria, S. R. Kuraria, R. N. Baghel and S. Thaker, J. Lumin., 2008, 128, 2038-2047.

7 Y. Xie and Z. Li, Mater. Chem. Front., 2020, 4, 317-331. 
8 L. Zhan, Z. Chen, S. Gong, Y. Xiang, F. Ni, X. Zeng, G. Xie and C. Yang, Angew. Chem., Int. Ed., 2019, 58, 17651-17655.

9 C. Wang, B. Xu, M. Li, Z. Chi, Y. Xie, Q. Li and Z. Li, Mater. Horiz., 2016, 3, 220-225.

10 Y. Xie and Z. Li, Chemistry, 2018, 4, 943-971.

11 H. Nakayama, J. i. Nishida, N. Takada, H. Sato and Y. Yamashita, Chem. Mater., 2012, 24, 671-676.

12 S. Xu, T. Liu, Y. Mu, Y. F. Wang, Z. Chi, C. C. Lo, S. Liu, Y. Zhang, A. Lien and J. Xu, Angew. Chem., Int. Ed., 2015, 54, 874-878.

13 J. Tu, Y. Fan, J. Wang, X. Li, F. Liu, M. Han, C. Wang, Q. Li and Z. Li, J. Mater. Chem. C, 2019, 7, 12256-12262.

14 Y. Xie, J. Tu, T. Zhang, J. Wang, Z. Xie, Z. Chi, Q. Peng and Z. Li, Chem. Commun., 2017, 53, 11330-11333.

15 J. Miao, Y. Zhang and M. Zhang, Mater. Chem. Front., 2021, 5, 6865-6872.

16 Y. Yu, Y. Fan, C. Wang, Y. Wei, Q. Liao, Q. Li and Z. Li, Mater. Chem. Front., 2021, 5, 817-824.

17 C. Wang, Y. Yu, Z. Chai, F. He, C. Wu, Y. Gong, M. Han, Q. Li and Z. Li, Mater. Chem. Front., 2019, 3, 32-38.

18 K. Duraimurugan, M. Harikrishnan, J. Madhavan, A. Siva, S. J. Lee, J. Theerthagiri and M. Y. Choi, Environ. Res., 2021, 194, 110741.

19 H. Liu, Y. Gu, Y. Dai, K. Wang, S. Zhang, G. Chen, B. Zou and B. Yang, J. Am. Chem. Soc., 2020, 142, 1153-1158.

20 Q. Sun, L. Tang, Z. Zhang, K. Zhang, Z. Xie, Z. Chi, H. Zhang and W. Yang, Chem. Commun., 2017, 54, 94-97.

21 Y. Yu, Y. Fan, C. Wang, Y. Wei, Q. Liao, Q. Li and Z. Li, J. Mater. Chem. C, 2019, 7, 13759-13763.

22 X. Lv, M. Sun, L. Xu, R. Wang, H. Zhou, Y. Pan, S. Zhang, Q. Sun, S. Xue and W. Yang, Chem. Sci., 2020, 11, 5058-5065.

23 A. Li, Z. Ma, J. Wu, P. Li, H. Wang, Y. Geng, S. Xu, B. Yang, H. Zhang, H. Cui and W. Xu, Adv. Opt. Mater., 2018, 6, 1700647.

24 H. Shu, H. Li, J. Rao, L. Chen, X. Wang, X. Wu, H. Tian, H. Tong and L. Wang, J. Mater. Chem. C, 2020, 8, 14360-14364.
$25 \mathrm{~J} . \mathrm{Xu}, \mathrm{A}$. Takai, Y. Kobayashi and M. Takeuchi, Chem. Commun., 2013, 49, 8447-8449.

26 J. Wang, Z. Chai, J. Wang, C. Wang, M. Han, Q. Liao, A. Huang, P. Lin, C. Li, Q. Li and Z. Li, Angew. Chem., Int. Ed., 2019, 58, 17297-17302.

27 J. Miao, CCDC 2048014: Experimental Crystal Structure Determination, 2020, DOI: $10.5517 /$ ccdc.csd.cc26r3z2.

28 J. Miao, CCDC 2048016: Experimental Crystal Structure Determination, 2020, DOI: 10.5517/ccdc.csd.cc26r415.

29 J. Miao, CCDC 2048015: Experimental Crystal Structure Determination, 2020, DOI: 10.5517/ccdc.csd.cc26r404.

30 J. Miao and CCDC 2089171, Experimental Crystal Structure Determination, 2021, DOI: 10.5517/ccdc.csd.cc283ymz.

31 Z. Monette, A. K. Kasar and P. L. Menezes, J. Mater. Sci., 2019, 30, 19675-19690.

32 J. Tu, F. Liu, J. Wang, X. Li, Y. Gong, Y. Fan, M. Han, Q. Li and Z. Li, ChemPhotoChem, 2019, 3, 133-137.

33 Q. Sun, K. Zhang, Z. Zhang, L. Tang, Z. Xie, Z. Chi, S. Xue, H. Zhang and W. Yang, Chem. Commun., 2018, 54, 8206-8209.

34 B. Xu, W. Li, J. He, S. Wu, Q. Zhu, Z. Yang, Y. C. Wu, Y. Zhang, C. Jin, P. Y. Lu, Z. Chi, S. Liu, J. Xu and M. R. Bryce, Chem. Sci., 2016, 7, 5307-5312.

35 Y. Jiang, X. Chang, W. Xie, G. Huang and B. S. Li, Mater. Chem. Front., 2021, 5, 885-892.

36 Y. Jiang, J. Wang, G. Huang, Z. Li, B. S. Li and B. Z. Tang, J. Mater. Chem. C, 2019, 7, 11790-11796.

37 C. Wang, Y. Yu, Y. Yuan, C. Ren, Q. Liao, J. Wang, Z. Chai, Q. Li and Z. Li, Matter, 2020, 2, 181-193.

38 F. Liu, J. Tu, X. Wang, J. Wang, Y. Gong, M. Han, X. Dang, Q. Liao, Q. Peng, Q. Li and Z. Li, Chem. Commun., 2018, 54, 5598-5601.

39 X.-J. Liu, H. Jiang, Y.-R. Jia and M. Xia, Dyes Pigm., 2020, 172, 107845.

40 H. Jiang, X.-J. Liu, R.-R. Jia, T.-H. Xu and M. Xia, $R S C A d v$, 2019, 9, 30381-30388.

41 M. Xia, G. Gao, H. Jiang, Y. Jia and X. Liu, Acta Chim. Sin., 2019, 77, 1194-1202. 